\title{
Comparative genomic analysis of the Tribolium immune system Zhen Zou ${ }^{\star *}$, Jay D Evans ${ }^{\dagger}$, Zhiqiang Lu* ${ }^{*}$, Picheng Zhao*, Michael Williams ${ }^{\ddagger}$, Niranji Sumathipala*, Charles Hetru ${ }^{\S}$, Dan Hultmark ${ }^{\ddagger}$ and Haobo Jiang ${ }^{{ }^{*}}$
}

Addresses: * Department of Entomology and Plant Pathology, Oklahoma State University, Stillwater, OK 74078, USA. ${ }^{+}$USDA-ARS Bee Research Laboratory, Beltsville, MD 20705, USA. *Umeå Centre for Molecular Pathogenesis, Umeå University, Umeå S-901 87, Sweden. §Institut Biol Moléc Cell, CNRS, Strasbourg 67084, France.

$\ltimes$ These authors contributed equally to this work.

Correspondence: Haobo Jiang. Email: haobo.jiang@okstate.edu

Published: 29 August 2007

Genome Biology 2007, 8:RI77 (doi:I0.1 I86/gb-2007-8-8-r I77)

The electronic version of this article is the complete one and can be found online at http://genomebiology.com/2007/8/8/RI77
Received: 8 August 2007

Revised: 8 August 2007

Accepted: 29 August 2007

(c) 2007 Zou et al.; licensee BioMed Central Ltd.

This is an open access article distributed under the terms of the Creative Commons Attribution License (http://creativecommons.org/licenses/by/2.0), which permits unrestricted use, distribution, and reproduction in any medium, provided the original work is properly cited.

\begin{abstract}
Background: Tribolium castaneum is a species of Coleoptera, the largest and most diverse order of all eukaryotes. Components of the innate immune system are hardly known in this insect, which is in a key phylogenetic position to inform us about genetic innovations accompanying the evolution of holometabolous insects. We have annotated immunity-related genes and compared them with homologous molecules from other species.

Results: Around 300 candidate defense proteins are identified based on sequence similarity to homologs known to participate in immune responses. In most cases, paralog counts are lower than those of Drosophila melanogaster or Anopheles gambiae but are substantially higher than those of Apis mellifera. The genome contains probable orthologs for nearly all members of the Toll, IMD, and JAK/STAT pathways. While total numbers of the clip-domain serine proteinases are approximately equal in the fly (29), mosquito (32) and beetle (30), lineage-specific expansion of the family is discovered in all three species. Sixteen of the thirty-one serpin genes form a large cluster in a 50 $\mathrm{kb}$ region that resulted from extensive gene duplications. Among the nine Toll-like proteins, four are orthologous to Drosophila Toll. The presence of scavenger receptors and other related proteins indicates a role of cellular responses in the entire system. The structures of some antimicrobial peptides drastically differ from those in other orders of insects.
\end{abstract}

Conclusion: A framework of information on Tribolium immunity is established, which may serve as a stepping stone for future genetic analyses of defense responses in a nondrosophiline genetic model insect.

\section{Background}

Tribolium beetles harbor a range of natural pathogens and parasites, from bacteria to fungi, microsporidians and tapeworms $[1,2]$. There is good evidence for genetic variation in resistance to the tapeworm and a linked cost of resistance in terms of growth and reproduction [3]. Cross-generational transfer of immune traits [4] may occur in Tenebrio molitor, a close relative of Tribolium castaneum. RNA interference 
experiments demonstrate that Tribolium laccase-2 is responsible for cuticle pigmentation and sclerotization [5]. While these observations are interesting, our knowledge of the genetic constituents of Tribolium immunity is almost blank at the cellular and molecular levels, in contrast to the vast amount of information regarding Drosophila melanogaster and Anopheles gambiae defense responses [6,7]. Given the high efficiency of RNA interference and powerful tools of molecular genetics [8], it is particularly appealing to use $T$. castaneum for the dissection of insect immune pathways. Acquired knowledge may be useful in controlling beetle pests that feed on crop plants or stored products.

In the broader field of beetle immunity, research has been focused mainly on two effector mechanisms, namely antimicrobial peptide synthesis and prophenoloxidase (proPO) activation [9]. Defensins, coleoptericins, cecropin and antifungal peptides have been isolated from coleopteran insects and characterized biochemically [10-12]. A homolog of human $\mathrm{NF}-\kappa \mathrm{B}$ (Allomyrina dichotoma Rel A) up-regulates the transcription of a coleoptericin gene [13]. Active phenoloxidase generates quinones for melanin formation, wound healing, and microbe killing. ProPO activation has been investigated in Holotrichia diomphalia [14-16]. ProPO activating factor 1 ( $H d$-PPAF1) cleaves proPO to generate active phenoloxidase in the presence of $H d$-PPAF2, the precursor of which is activated by $H d$-PPAF3 via limited proteolysis. While all these PPAFs contain an amino-terminal clip domain, PPAF2 (in contrast to PPAF1 or PPAF3) does not have catalytic activity since its carboxy-terminal serine proteinase-like domain lacks the active site serine. A $43 \mathrm{kDa}$ inhibitor down-regulates the melanization response in $H$. diomphalia [17].

To date, components of the innate immune system are hardly known in T. castaneum and neither is it clear how they differ from homologous molecules in the honeybee, mosquito or fruitfly $[6,7,18]$. This lack of knowledge does not seem to reconcile with the critical phylogenetic position of this coleopteran species, which should inform us a lot about genetic variations in the evolution of holometabolous insects. Information regarding defense responses in T. castaneum, a member of the largest and most diverse order of eukaryotes, is highly desirable for the biological control of crop pests and disease vectors. Consequently, we have used its newly available genome assembly to annotate immunity-related genes and analyze their phylogenetic relationships with homologous sequences from other insects. In this comparative overview of the Tribolium defense system, we describe plausible immune pathway models and present information regarding the molecular evolution of innate immunity in holometabolous species.

\section{Results and discussion \\ Overview of the Tribolium immune system}

$T$. castaneum has a sizable repertoire of immune proteins predicted to participate in various humoral and cellular responses against wounding or infection (Additional data file 1). Like other insects $[6,7,19]$, cuticle and epithelia lining its body surfaces, tracheae and alimentary tract may serve as a physiochemical barrier and local molecular defense by producing antimicrobial peptides and reactive oxygen/nitrogen species (ROS/RNS). While this line of defense may block most pathogens, others enter the hemocoel where a coordinated acute-phase reaction could occur to immobilize and kill the opportunists. This reaction, including phagocytosis, encapsulation, coagulation and melanization, is probably mediated by hemocytes and molecules constitutively present in the circulation. These first responders may not only control minor infections but also call fat body and hematopoietic tissues for secondary responses if necessary. At the molecular level, the following events should take place in all insects, including the beetle: recognition of invading organisms by plasma proteins or cell surface receptors, extra- and intracellular signal transduction and modulation, transcriptional regulation of immunity-related genes, as well as controlled release of defense molecules.

\section{Pathogen recognition}

Peptidoglycan recognition proteins (PGRPs) serve as an important surveillance mechanism for microbial infection by binding to Lys- and diaminopimelate-type peptidoglycans of walled bacteria [20]. Some Drosophila PGRPs (for example, LC and SA) are responsible for cell-mediated or plasma-based pathogen recognition; others (that is, LB and $\mathrm{SB}$ ) may hydrolyze peptidoglycans to turn on/off immune responses [21,22]. In $T$. castaneum, PGRP-LA, -LC and -LD contain a transmembrane segment; PGRP-SA and -SB are probably secreted; PGRP-LE (without a signal peptide or transmembrane region) may exist in cytoplasm or enter the plasma via a nonclassical secretory pathway. Bootstrap analysis and domain organization clearly indicate that Tribolium and Drosophila PGRP-LEs are orthologs - so far no PGRP-LE has been identified in Anopheles, Bombyx or Apis. Other orthologous relationships (for example, TcPGRP-LC and AmPGRPLC) are also supported by the phylogenetic analysis (Figure 1). The beetle and mosquito PGRP-LA genes encode two alternative splice forms (PGRP-LAa and -LAb). Like Drosophila and Anopheles, Tribolium PGRP-LA and -LC genes are next to each other in the same cluster. Most of the beetle PGRPs resulted from ancient family diversification that occurred before the emergence of holometabolous insects. In contrast, gene duplication occurred several times in the lineages of mosquito and fly (Figure 1).

Multiple sequence alignment suggests that $\beta-1,3$-glucan-recognition proteins ( $\beta$ GRPs) and Gram-negative binding proteins (GNBPs) are descendents of invertebrate $\beta-1,3^{-}$ glucanases [23]. Lacking one or more of the catalytic residues, 


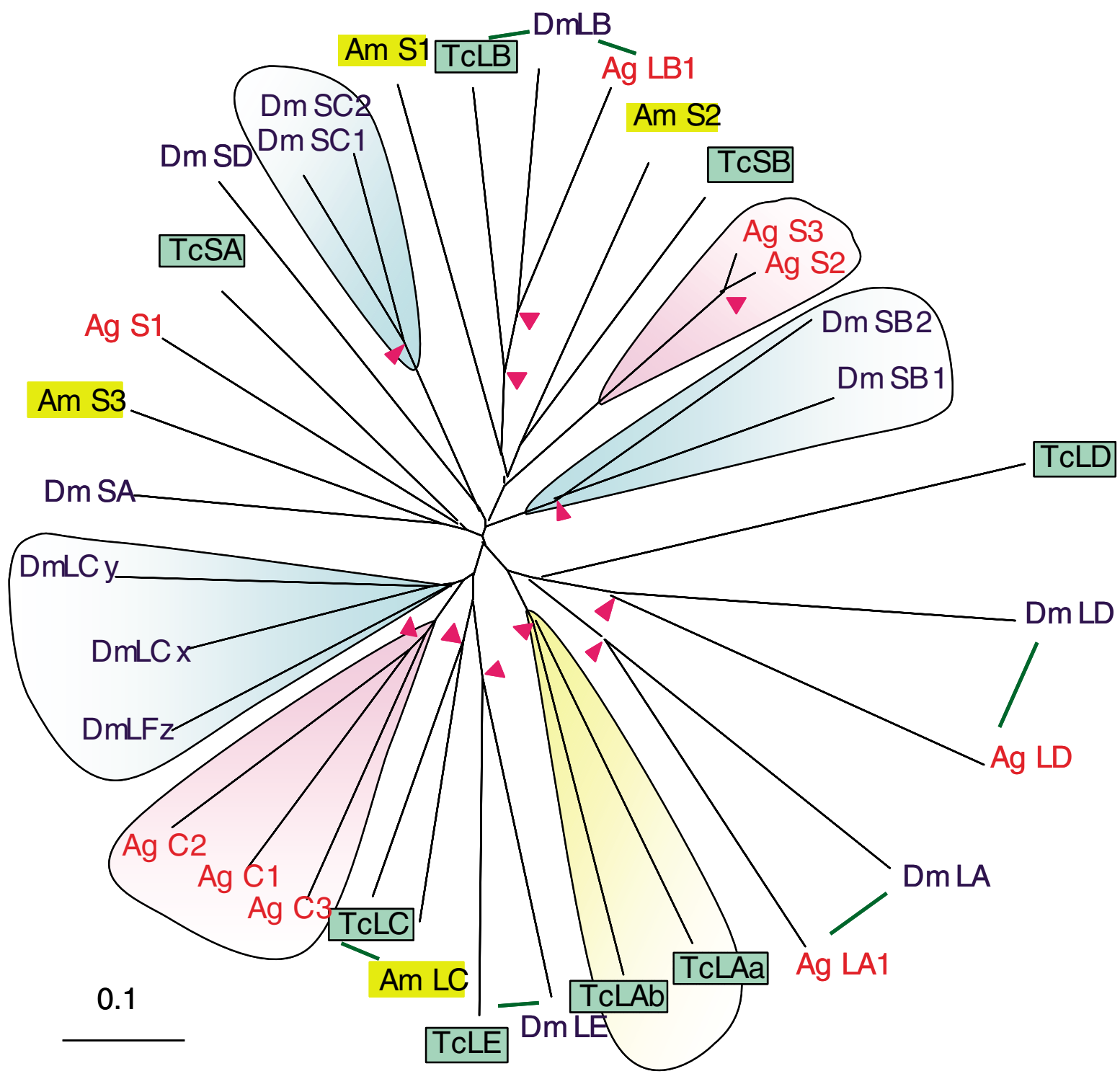

Figure I

Peptidoglycan recognition proteins. The amino acid sequences from eight Tribolium (Tc), thirteen Drosophila (Dm), nine Anopheles (Ag), and four Apis (Am) PGRPs are examined. The phylogenetic tree shows family expansion in Tribolium (shaded yellow), Anopheles (shaded pink) and Drosophila (shaded blue). TCPGRP-LA, -LC and -LD contain a transmembrane domain whereas TCPGRP-SA and -SB have a signal peptide for secretion. Pink arrowheads at nodes denote bootstrap values greater than 800 from I,000 trials. The putative I:I or I:I:I orthologs are connected by green lines. TCPGRP-LB and -SB contain the key residues for an amidase activity.

these homologous molecules do not possess any hydrolytic activity. They are widespread in arthropods and act in part to recognize microbial cell wall components such as $\beta-1,3-$ glucan, lipoteichoic acid or lipopolysaccharide. We have identified three $\beta$ GRPs in T. castaneum. Tc- $\beta$ GRP1 and AgGNBP$\mathrm{B} 1$ through -B5 are closely related and represent a young lineage, whereas Tc- $\beta$ GRP2 and Tc- $\beta$ GRP 3 belong to an ancient group that arose before the radiation of holometabolous insects (Additional data file 2). Since Drosophila has no $\beta$ GRP-B and Anopheles has five, the presence of a single gene (encoding Tc- $\beta$ GRP1) in the beetle can be useful for elucidating function of this orthologous group. In addition to the glucanase-like domain, members of the second group contain an amino-terminal extension of about 100 residues. In Bombyx 
mori $\beta$ GRP, this region recognizes $\beta$-1,3-glucan also [24]. $M$. sexta $\beta$ GRP2 binds to insoluble $\beta$-1,3-glucan and triggers a serine proteinase cascade for proPO activation [25].

C-type lectins (CTLs) comprise a wide variety of soluble and membrane-bound proteins that associate with carbohydrates in a $\mathrm{Ca}^{2+}$-dependent manner [26]. Some insect CTLs recognize microorganisms and enhance their clearance by hemocytes [19]. Gene duplication and sequence divergence, particularly in the sugar-interacting residues, lead to a broad spectrum of binding specificities for mannose, galactose and other sugar moieties. These proteins associate with microbes and hemocytes to form nodules [27] and stimulate melanization response [28]. T. castaneum encodes sixteen CTLs: ten (Tc-CTL1, 2, 4 through 10, and 13) with a single carbohydrate recognition domain and one (Tc-CTL3) with two. Five other proteins, tentatively named Tc-CTL11, 12, 14, 15 and 16, contain a CTL domain, a transmembrane region (except for $T c$ CTL11), and other structural modules: CTL11 has three CUB and three EGF; CTL12 has six Ig and three FN3; CTL14 has one $\mathrm{LDL}_{\mathrm{r}} \mathrm{A}$, three CUB, ten Sushi, nineteen EGF, two discoidin, one laminin G and one hyalin repeat; CTL15 has one FTP, eleven Sushi and two EFh; CTL16 has one FTP and four Sushi. While lineage-specific expansion of the gene family is remarkable in D. melanogaster and A. gambiae [29], we have not found any evidence for that in T. castaneum (or A. mellifera): Tc-CTL1, 2, 5, 6, 8, 9, 12 through 16 have clear orthologs in the other insect species whereas Tc-CTL7, 10 and 11 are deeply rooted (Additional data file 3 ).

Galectins are $\beta$-galactoside recognition proteins with significant sequence similarity in their carbohydrate-binding sites characteristic of the family. Drosophila DL1 binds to E. coli and Erwinia chrysanthemi [30]. Leishmania uses a sandfly galectin as a receptor for specific binding to the insect midgut [31]. Tc-galectin1 has two carbohydrate recognition domains; Tc-galectin2 and 3 are orthologous to Am-galectin1 and 2, respectively (Additional data file 4).

All fibrinogen-related proteins (FREPs) contain a carboxyterminal fibrinogen-like domain associated with different amino-terminal regions. In mammals, three classes of FREPs have been identified: ficolin, tenascins, and microfibril-associated proteins [32]. They take part in phagocytosis, wound repair, and cellular adhesion [33]. In invertebrates, FREPs are involved in cell-cell interaction, bacterial recognition, and antimicrobial responses [34-36]. The Tribolium genome contains seven FREP genes, which fall into three groups (Additional data file 5): the expansion of group I yielded four family members: Tc-FREP1 through 4. Sitting next to each other on chromosome 3 , these beetle genes encode polypeptides most similar to angiopoietin-like proteins. During angiogenesis, the human plasma proteins interact with tyrosine kinase receptors (for example, Tie) and lead to wound repair and tissue regeneration [37]. In group II, Tc-FREP 5 is orthologous to $D m$-scabrous, which is required for Notch signaling during tissue differentiation [38]. Interestingly, Notch is also needed for proper differentiation of Drosophila hemocytes [39]. Group III includes Tc-FREP6, Tc-FREP7, Ag-FREP9 and Dm-CG9593. No major expansion has occurred in the beetle or honeybee, in sharp contrast to the situations in the fly and mosquitoes - there are 61 FREP genes in the A. gambiae genome [29].

Thioester-containing proteins (TEPs), initially identified in D. melanogaster [39], contain a sequence motif (GCGEQ) commonly found in members of the complement $\mathrm{C}_{3} / \alpha_{2}$ macroglobulin superfamily. After cleavage activation, some TEPs use the metastable thioester bond between the cysteine and glutamine residues to covalently attach to pathogens and 'mark' them for clearance by phagocytosis [40]. One of the 15 TEPs in Anopheles, $A g$-TEP1, plays a key role in the host response against Plasmodium infection and ten other $\mathrm{Ag}$ TEPs are results of extensive gene duplications. This kind of family expansion did not happen in the beetle (or bee): Tribolium encodes four TEPs, perhaps for different physiological purposes. Our phylogenetic analysis supports the following orthologous relationships: TcA-AmA-Ag13-Dm6, TcB-AmB$A g 15-D m_{3}$, and $T c \mathrm{C}-A m \mathrm{C}$ (Additional data file 6).

\section{Extracellular signal transduction and modulation}

Similar to the alternative and lectin pathways for activation of human complements, insect plasma factors play critical roles in pathogen detection, signal relaying/tuning, and execution mechanisms. Serine proteinases (SPs) and their noncatalytic homologs (SPHs) are actively involved in these processes. Some SPs are robust enzymes that hydrolyze dietary proteins; others are delicate and specific - they cleave a single peptide bond in the protein substrates. The latter interact among themselves and with pathogen recognition proteins to mediate local responses against nonself. The specificity of such molecular interactions could be enhanced by SPHs, adaptor proteins that lack proteolytic activity due to substitution of the catalytic triad residues. SPs and SPHs constitute one of the largest protein families in insects [29,41,42]. We have identified 103 SP genes and 65 SPH genes in the Tribolium genome, 77 of which encode polypeptides with a SP or SP-like domain and other structural modules. These include thirty SPs and eighteen SPHs containing one or more regulatory clip domains. Clip-domain SPs, and occasionally clip-domain SPHs, act in the final steps of arthropod SP pathways [43]. Other recognition/regulation modules (for example, $\mathrm{LDL}_{\mathrm{r}} \mathrm{A}$, Sushi, CUB and CTL) also exist in long SPs (>300 residues), some of which act in the beginning steps of SP pathways.

T. castaneum clip-domain proteins are divided into four subfamilies (Figure 2). Even though the catalytic or proteinaselike domains used for comparison were similar in length and sequence, we found subfamily A is composed of SPHs solely whereas subfamilies B, C and D comprise SPs mainly. Apparently, it is easier for SPs to lose activity and become SPHs during evolution than for SPHs to regain catalytic activity. The 
four groups of SP-related genes may represent lineages derived from ancient evolutionary events since similar subfamilies also exist in Anopheles and Drosophila. Moreover, expansion of individual subfamilies must have occurred several times to account for the gene clusters observed in the Tribolium genome (Figure 2). Evidence for lineage-specific gene duplication and movement is also present in the mosquito and fly genomes $[29,41]$. Based on the results of genetic/biochemical analysis performed in other insects [14-16,19,44,45] and sequence similarity, we are able to predict the physiological functions for some Tribolium clip-domain SPs and SPHs during proPO activation and spätzle processing. For instance, Tc-SPH2, $\mathrm{SPH}_{3}$ or $\mathrm{SPH}_{4}$ (similar to $\mathrm{Hd}$-PPAF2) may serve as a cofactor for $\mathrm{Tc}-\mathrm{SP} 7, \mathrm{SP} 8$ or SP10 (putative proPO activating proteinases); Tc-SP44 or SP66 may function like Drosophila persephone [46]; Tc-SP136 or SP138 may activate spätzle precursors by limited proteolysis [44,45].

Most members of the serpin superfamily are irreversible inhibitors of SPs and, by forming covalent complexes with diffusing proteinases, they ensure a transient, focused defense response [47]. There are totally 31 serpin genes in $T$. castaneum, more than that in D. melanogaster (28), A. gambiae (14) or A. mellifera (7). This number increase is mainly caused by a recent family explosion at a specific genomic location - we have identified a cluster of 16 serpin genes in a small region of 50 kilobases on chromosome 8 . These closely related genes constitute a single clade in the phylogenetic tree (Figure 3). Sequence divergence, especially in the reactive site loop region, is anticipated to alleviate the selection pressure imposed by the SP family expansion (Figure 2). Exon duplication and alternative splicing, found in 4 of the 31 serpin genes, also generate sequence diversity and inhibitory selectivity.

\section{Intracellular signal pathways and their regulation}

Drosophila Toll is a transmembrane protein that binds spätzle and relays developmental and immune signals [48]. Resulting from ancient family expansion, a total of five spätzle homologs and eight Toll-like receptors are present in the fly. There are seven Tribolium genes coding for spätzle-like proteins, most of which have putative orthologs in Drosophila and Anopheles (Additional data file 7). Like their ligands, Toll-like proteins have also experienced major family expansion and sequence divergence. The receptors are separated into two clusters, with the fly and beetle Toll-9 located near the tree center (Figure 4). While Toll-6, -7, -8 and -10 from different insect species constitute tight orthologous groups in one cluster, lineage-specific gene duplications have given rise to Drosophila Toll-3 and -4, Anopheles Toll-1 and 5, as well as Tribolium Toll-1 through -4. Located on the same branch with Drosophila Toll, the four Tribolium receptors could play different yet complementary roles in the beetle defense and development. In addition, we have identified eight MD2-related genes in the beetle. Mammalian MD2, Toll-like receptor-4 and CD14 form a complex that recognizes lipopolysaccharides [49]. The Anopheles MD2-like receptor regulates the specificity of resistance against Plasmodium berghei $[50]$.

Contrary to the ligand-receptor diversification, components of the intracellular pathway appear to be highly conserved in insects studied so far (Figure 5a). In Drosophila, multimerization of Toll receptors caused by spätzle binding leads to the association of dMyD88, Tube, Pelle, Pellino and dTRAF6 [51]. With 1:1 orthologs identified in the beetle (as well as the other insects with known genomes), we postulate that a similar protein complex also forms to phosphorylate a cactus-like molecule (Tc02003). The modified substrate protein then dissociates from its partner (Tc07697 or Tco896), allowing the Rel transcription factors to translocate into the nucleus and activate effector genes (for example, antimicrobial peptides). Functional tests are required to verify the suggested roles of individual components during defense and development in the beetle.

The IMD pathway is critical for fighting certain Gram-negative bacteria in Drosophila. Upon recognition of diaminopimelate-peptidoglycan by PGRPs, the 'danger' signal is transduced into the cell through IMD (Figure $5 \mathrm{~b}$ ). IMD contains a death domain that recruits dFADD (dTAK1 activator) and Dredd (a caspase). Active dTAK1 is a protein kinase that triggers the JNK pathway (through Hep, Basket, Jra and Kay) and Relish phosphorylation (through Ird5 and Kenny). The presence of $1: 1$ orthologs in $T$. castaneum strongly suggests that IMD-mediated immunity is conserved in the beetle. Furthermore, the modulation of these pathways may also resemble each other - we have identified putative 1:1 orthologs of IAP2, Tab2 and caspar in the Tribolium genome (Figure 5b).

The transcription of Drosophila TEPs and some other immune molecules is under the control of the JAK-STAT pathway [52]. This pathway, triggered by a cytokine-like molecule, Upd3, promotes phagocytosis and participates in an antiviral response. Based on sequence similarity, we predict that the conserved signaling pathway in the beetle is composed of the orthologs of Dm-Domeless, Hopscotch and STAT92 (Figure 5c). However, we have not identified any ortholog of Dm-upd, upd2, or upd3, possibly due to high sequence variation in the cytokine-like proteins.

\section{Execution mechanisms}

Phenoloxidases are copper-containing enzymes involved in multiple steps of several immune responses against pathogens and parasites (that is, clot reinforcement, melanin formation, ROS/RNS generation, and microbe killing) [53]. Synthesized and released as an inactive zymogen, proPO requires a SP cascade for its cleavage activation. SPHs and serpins ensure that the proteolytic activation occurs locally and transiently in response to infection. We have identified three proPO genes in the Tribolium genome, designated proPO1, 2 and 3. Tc-proPO2 and proPO3 are 98.8\% identical in nucleotide sequence and $99.6 \%$ identical in amino acid 


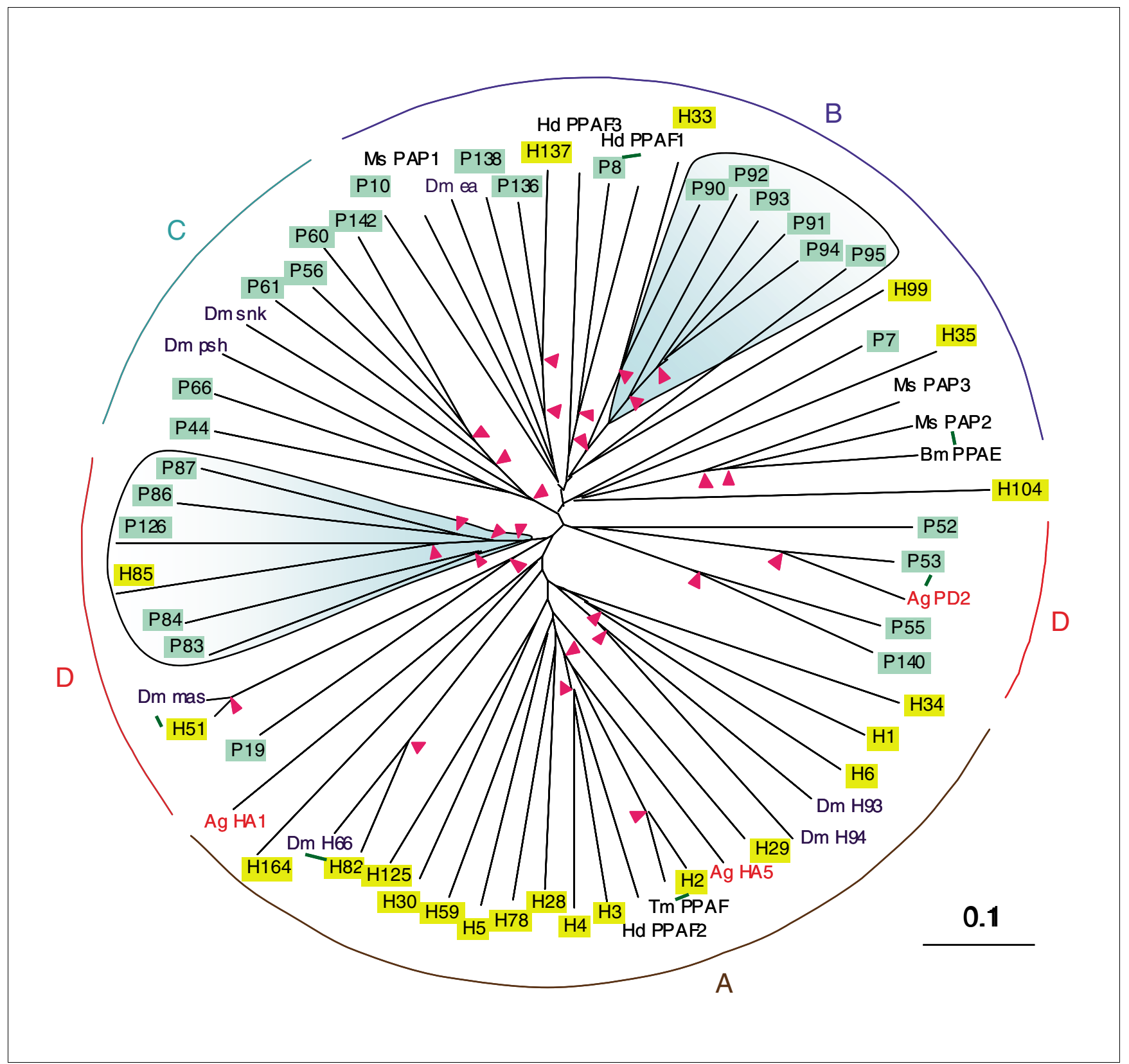

Figure 2

Expansion of the clip-domain family of SPs and SPHs in the T. castaneum genome. The catalytic and proteinase-like domains in the 49 Tribolium sequences are compared with those in 7 Drosophila (Dm), 3 Anopheles (Ag), 3 Holotrichia $(\mathrm{Hd})$, I Tenebrio (Tm), I Bombyx (Bm) and 3 Manduca (Ms) SP-related proteins. The tree is divided to four clades (A to D). While clade A contains SPHs (yellow) only, the other three are mainly SPs (green). Region D, split into two parts, is intact when all the group D clip-domain proteins from Drosophila and Anopheles are included in the analysis (data not shown). Pink arrowheads at nodes indicate bootstrap values greater than 800 from I,000 trials. The putative ortholog pairs are connected with green bars. Other than the shown ones (shaded blue, excluding SPI26), there are four clusters of clip-domain SP/SPH genes in the genome: (SP)HI through H6, (S)P7 through PI0, H28 and H29, PI 35 through PI39. Some of them (P9, PI35 and PI39) have no clip domain and, thus, are not shown in the figure.

sequence. In the aligned coding regions (2,052 nucleotides long), 21 of the 24 substitutions are synonymous, corresponding to 0.0102 changes/site. These two genes are $530 \mathrm{~kb}$ apart and their aligned intron regions are $88.5 \%$ identical. Using the relative rate of nucleotide substitutions derived from an analysis of Drosophila alcohol dehydrogenase genes [54], we estimate that $T c$-proPO2 and $T c$-proPO 3 arose by gene duplication approximately o.6 million years ago. The phylogenetic analysis suggests that such evolutionary events are sporadic for this family: the total numbers of proPO genes in different insect species did not change significantly, except for the malaria mosquito (Additional data file 8). Of the nine $\mathrm{Ag}$ - 

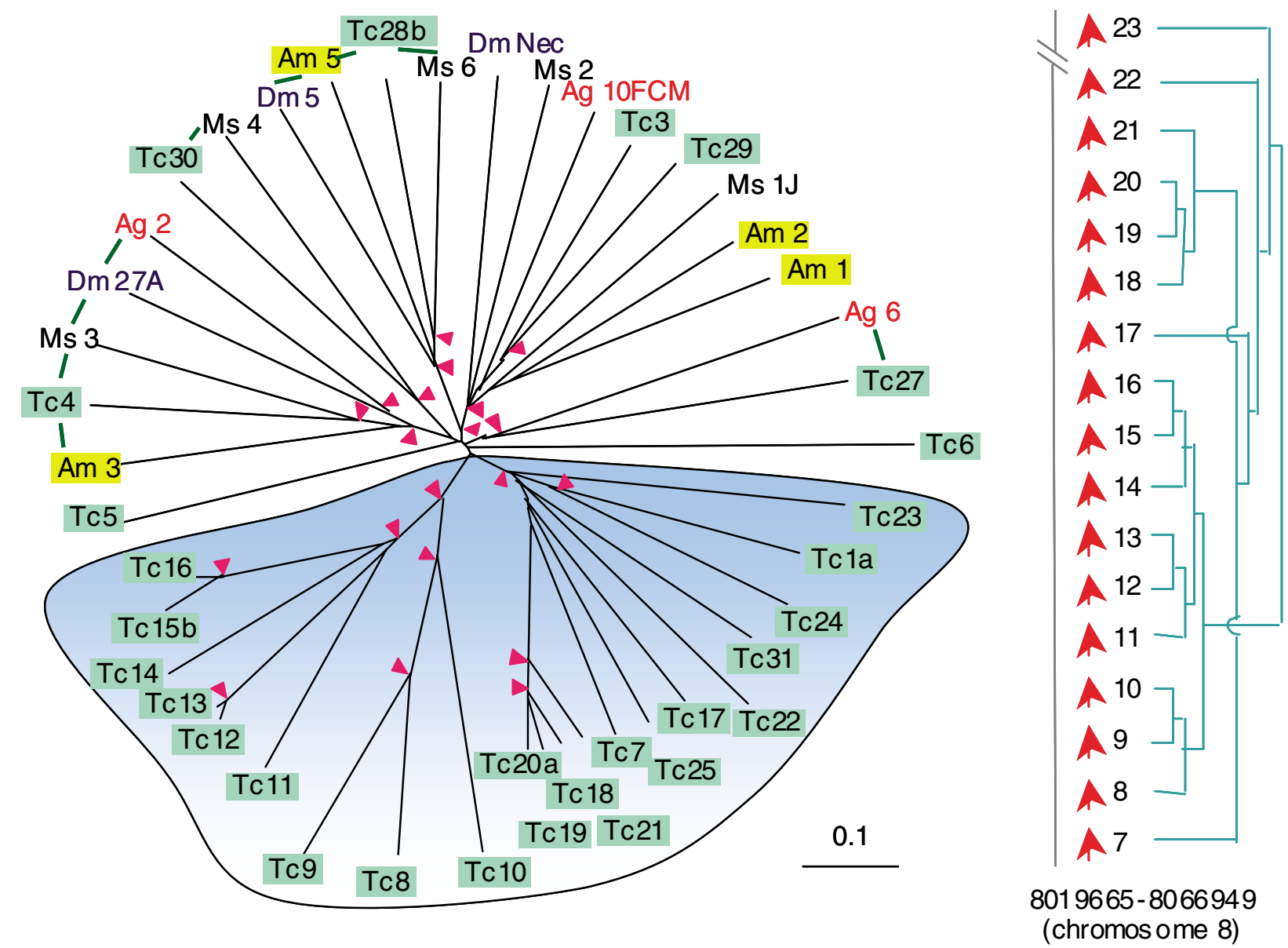

Figure 3

A major family expansion of Tribolium serpins and their phylogenetic relationships with the serpins from other insect species. The sequences of 29 Tribolium (Tc), 3 Drosophila (Dm), 3 Anopheles (Ag), 4 Apis (Am) and 5 Manduca (Ms) serpins are compared. Tribolium serpin2 (758 residues) and serpin26 (568 residues), much longer than a typical serpin (40-50 kDa), are excluded from the analysis. For simplicity, Tribolium serpins Ib, $15 \mathrm{a}, 20 \mathrm{~b}$ and $28 \mathrm{a}$ are also eliminated because they are products of alternative splicing of the genes Ia, 15b, 20a and 28b, which differ only in the region coding for reactive site loop. As shown in the tree (left panel), extensive expansion gives rise to this group of highly similar genes (shaded blue) located in a small chromosomal region (right panel). Pink arrowheads at nodes denote bootstrap values greater than 800 for I,000 trials. Putative I:I, I:I:I or I:I:I:I orthologous relationship is indicated by green bars connecting the group members.

proPO genes, eight arose from gene expansion that occurred early in the mosquito lineage [29], some of which encode phenoloxidases for melanization.

Local production of free radicals is a critical component of the acute-phase oxidative defense, involving nitric oxide synthase, NADPH oxidase, peroxidase, phenoloxidase and other enzymes [53,55]. Due to the cytotoxicity of ROS and RNS, their conversion and concentrations must be tightly regulated by superoxide dismutases (SODs), glutathione oxidases (GTXs), catalases, thioredoxins, thioredoxin reductases, melanin intermediates, and certain metal ions. Changes in the free radical levels by gene mutation or knock-down affect the fecundity and antimalarial response of the mosquito [56]. We have annotated some of these genes in Tribolium, including peroxidases, GTXs, SODs, peroxiredoxins (TPXs) and catalases. T. castaneum GTX1-GTX2 and TPX2-TPX6 gene pairs are results of recent gene duplications, whereas several orthologous relationships have been identified in the SOD and TPX families in the phylogenetic analysis (Additional data file 9).

Coleopteran species have been explored at the biochemical level for various antimicrobial peptides (AMPs) [57]. While defensins are present in all insects studied, coleoptericins are related to the attacin/diptericin family of glycine-rich anti- 


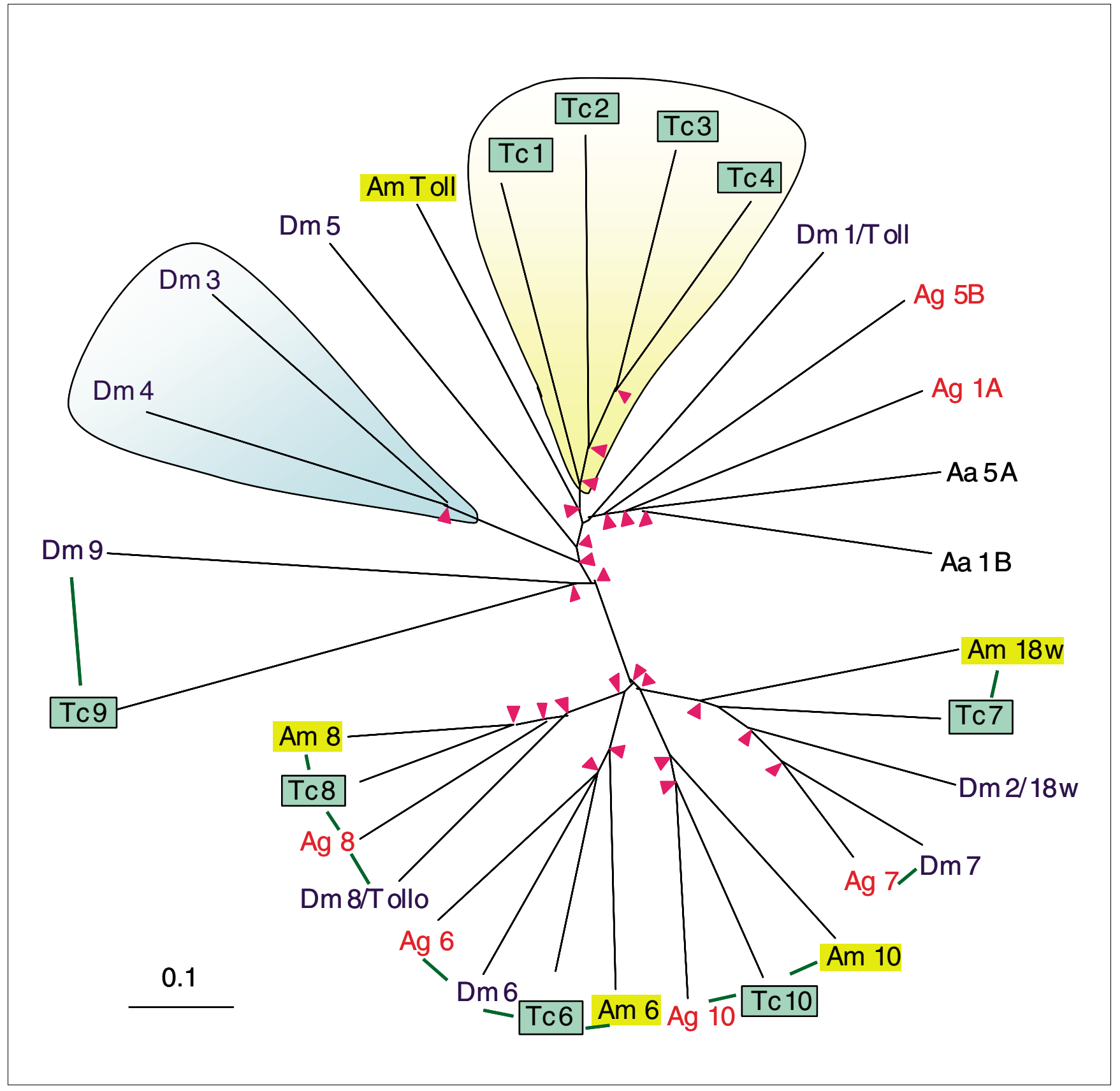

Figure 4

Phylogenetic relationships of Toll-like receptors from five insect species. The sequences of nine Tribolium (Tc), nine Drosophila (Dm), six Anopheles (Ag), five Apis (Am), and two Aedes (Aa) Toll-related proteins are compared. Species-specific family expansion is shaded yellow for Tribolium and blue for Drosophila. Nodes with pink arrowheads have bootstrap values exceeding 800 from I,000 trials, and green lines connect putative orthologs with I:I, I:I:I or I:I:I:I relationship. Note that TcToll-9 does not have a Toll/interleukin I receptor domain.

bacterial peptides in lepidopteran and dipteran species [58]. Four defensin genes are detected in the Tribolium genome, three of which are found in a branch containing only coleopteran insects (Figure 6). Tc-defensin 4 is in a miscellaneous group containing Odonata, Lepidoptera and Arachnida species. Interestingly, defensins of three other coleopteran insects are in the same branch with the hymenopteran ones.
Like the beetle defensins, coleoptericins belong to two phylogenetic groups, with the same separation of species in each group.

With the genome sequence available, we are able to use the other AMP sequences to identify homologous genes that are not specified in beetles. Cecropins were mostly identified in 
(a)

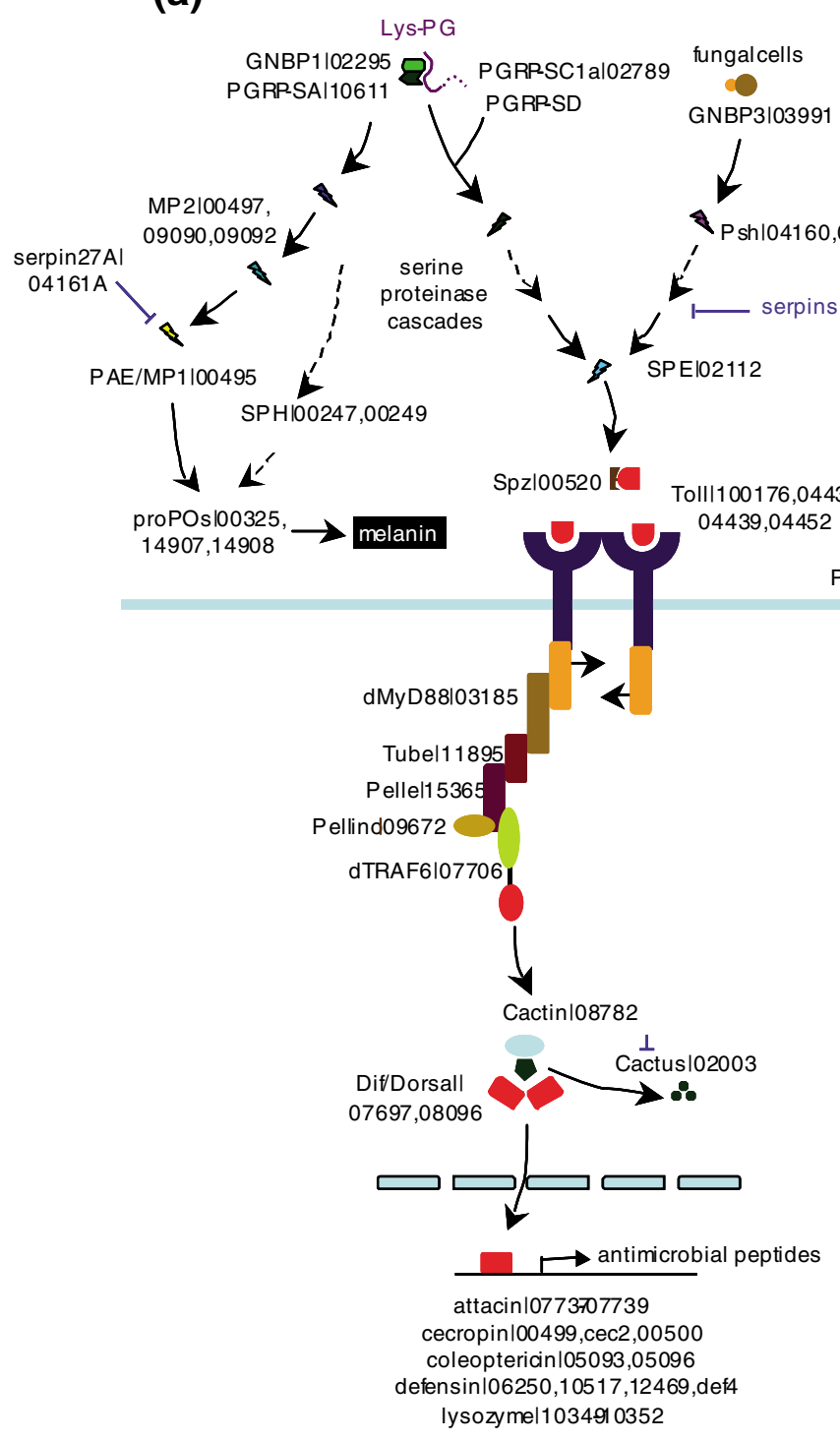

(b)

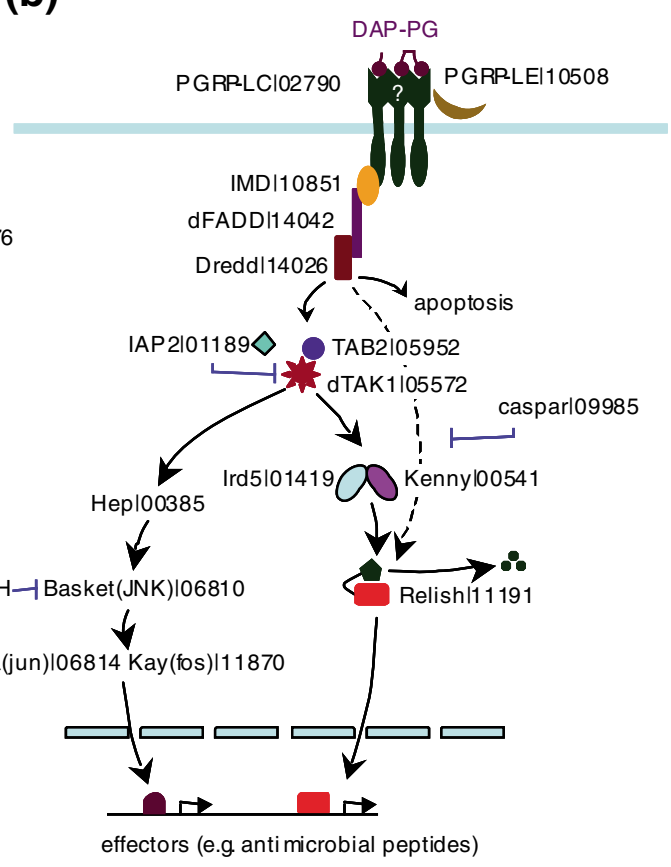

(c)

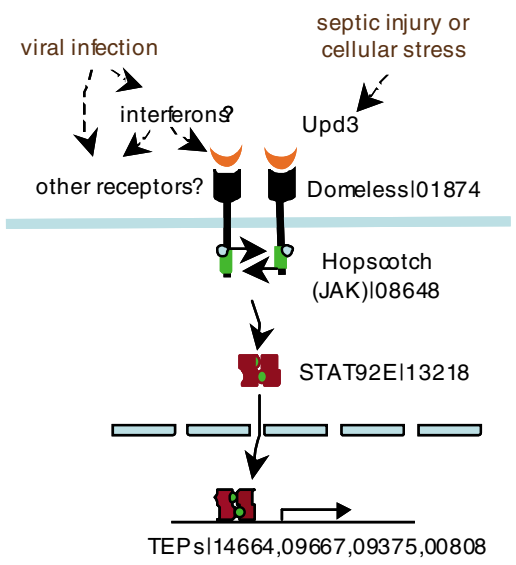

Figure 5

Schematic drawing of the immune signaling pathways in Drosophila and Tribolium. (a) Extracellular serine proteinase pathways for proPO and Spätzle activation as well as the intracellular Toll pathway for antimicrobial peptide production. (b) IMD pathway and JNK branch for induced synthesis of immune responsive effectors. (c) JAK-STAT pathway for transcription activation of defense genes (for example, TEPs). Components of the putative pathways from $T$. castaneum are predicted based on sequence similarity. The Drosophila gene names are followed by GLEAN numbers of their beetle orthologs (or paralogs in some cases).

moths and flies - there was only one report on cecropin from a coleopteran species, Acalolepta luxuriosa [11]. In Tribolium, we find a single close homolog of the Acalolepta cecropin, although a frame shift in a run of seven adenosines indicate that this is a pseudogene (Tco0499). Closely linked to Tcoo499 on chromosome 2 are two genes that encode cecropin-related peptides of unusual structure, with proline- and tyrosine-rich carboxy-terminal extensions ( $T c$-cecropin2 and Tc00500). These observations indicate that cecropins may widely exist in beetles. Attacins were found only in lepidopteran and dipteran species. We have identified a cluster of three attacin genes (Tc07737-07739) on Tribolium chromosome 4. Although we failed to identify a Drosomycin homolog in the beetle, our search resulted in a low-score hit of a 


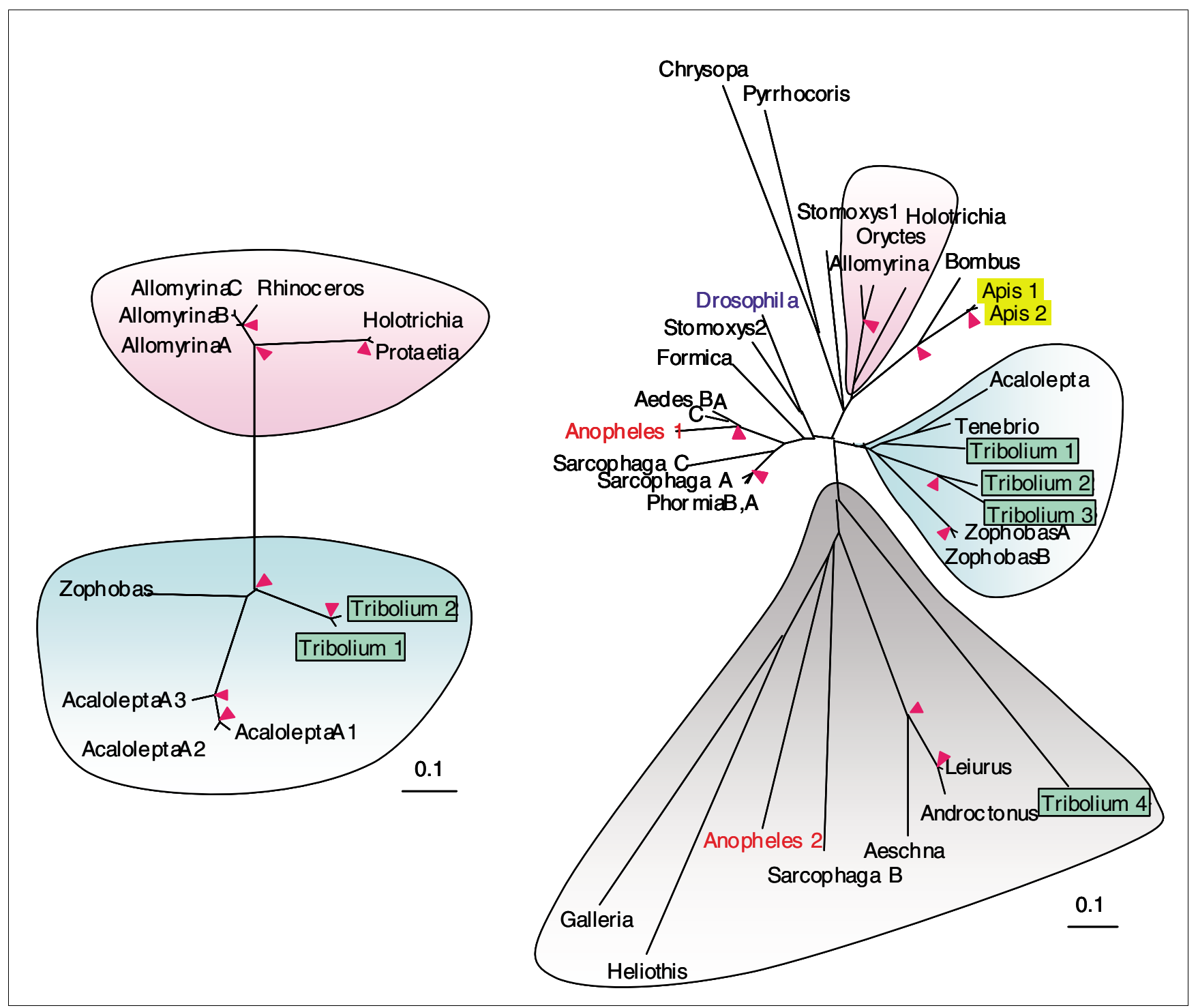

Figure 6

Evolutionary relationships of the coleoptericins (left panel) and defensins (right panel). The alignment of mature antimicrobial peptide sequences is used to build the phylogenetic trees on which their genus names are indicated. The beetle coleoptericins and defensins are divided into two subgroups (shaded blue and pink), whereas the more primitive defensins (shaded grey) are found in many arthropod species. Note that the blue clades include Acalolepta, Tribolium and Zophobas whereas the pink clades both contain Allomyrina and Holotrichia. Pink arrowheads at nodes denote bootstrap values greater than 800 from I,000 trials. This analysis uses sequences from the orders of Coleoptera (Acalolepta, Allomyrina, Holotrichia, Oryctes, Protaetia, Rhinoceros, Tenebrio, Tribolium, Zophobas), Diptera (Aedes, Anopheles, Drosophila, Phormia, Sarcophaga, Stomoxys), Lepidoptera (Galleria, Heliothis), Hemiptera (Pyrrhocoris), Hymenoptera (Apis, Bombus, Formic), Neuroptera (Chrysopa), Ordonata (Aeschna) and Scopiones (Androctonus, Leiurus).

cysteine-rich sequence. The corresponding gene (Tc11324) encodes a 104 residue polypeptide containing 2 whey acidic protein motifs. While mammalian proteins with this motif possess antibacterial activities [59], expression and biochemical analyses are needed to test if the Tribolium protein has a similar function. Due to the presence of species-specific AMPs and severe sequence diversity of these molecules, our homology-based search has probably missed some AMP genes. Should there be a thorough exploration by sequence similarity, biochemical separation and activity assays (not only against Gram-positive and Gram-negative bacteria, but also against yeasts and filamentous fungi), we expect the total number of AMPs (currently 12) in T. castaneum may approach that (20) in D. melanogaster. In addition to these, we have found a cluster of four lysozyme genes in the Tribolium genome (Additional data file 10). Similar but independent family growths have occurred in different insect groups, giving rise to thirteen such genes in Drosophila, eight in Anopheles, three in Apis, and four in Tribolium.

Cellular responses (that is, phagocytosis, nodulation and encapsulation) play key roles in the insect innate immunity 
[6o]. In the past few years, breakthroughs have been made in the molecular dissection of these processes [61]. Drosophila Peste, Eater, scavenger receptor (SR)-CI, Dscam, TEPs, and PGRP-SC1a seem to be implicated in the phagocytosis. Multiple SR-B genes are present in the Tribolium (16), Drosophila (12) and Anopheles (16) genomes, indicative of important functions of the subfamily. A phylogenetic analysis of the SRBs (Figure 7) demonstrates that nearly half of the members arose from ancient gene duplication events - we can easily identify orthologs from different insect species. More recent family expansions in the mosquito [29] and beetle account for the other half of the subfamily. There are two SR-B gene clusters in the Tribolium genome, one of which (TcSR-B14, -B15 and -B16) is located in the same branch containing Dm-peste. In addition to SR-Bs, Drosophila Nimrods are also involved in cellular responses [62]. The plasmatocyte-specific NimC1 directly participates in the phagocytosis of bacteria. For Tribolium, all three subclasses are represented: NimA, NimB and NimC, just like in the fly, mosquito and bee. However, unlike the other insects, the syntenic relationship is broken up in the beetle NimC homologs: the two NimC paralogs (Tc02053 and Tc15258) are not closely linked to the NimA and NimB homologs (Tc11427 and Tc11428). In the other insects, the order of nimA, nimB and nimC genes is well conserved.

\section{Expression analysis}

One characteristic of the innate immune system is that some of its components are transcriptionally up-regulated after a microbial challenge. To acquire evidence that the genes we annotated are involved in defense responses, we have exposed the adult beetles to E. coli, Micrococcus luteus, Candida albicans or Saccharomyces cerevisiae cells and isolated total RNA from the control and treated insects for expression analysis. Real-time PCR experiments indicated that transcript levels of some genes dramatically changed (Figure 8). TcPGRP-SA and TcPGRP-SB mRNA became more abundant after the bacterial infection, whereas the increase was much less significant for TcPGRP-LA, -LE, galectin1 or TEP-C after the $C$. albicans or M. luteus treatment. Following the Grampositive bacterial or fungal challenge, we detected some elevations in Tc-cSP66, serpin29 and serpin3o transcripts.

Transcriptional regulation is not limited to pattern recognition molecules or extracellular signal mediators/modulators: we detected differential expression of ligand and their receptors (for example, Tc-spätzle1, Toll-1 through Toll-4, and IMD). mRNA level changes for the latter genes were small except for IMD (Figure 8). Toll-3 and Toll-4 induction after the $C$. albicans or M. luteus challenge was apparent, although not as notable as IMD. The subtle changes in Toll-1 transcript levels were somewhat different from those of Toll-2, -3 and 4 , indicating that there could be functional differences and overlaps in antimicrobial responses for these closely related receptors (Figure 4).
We have also examined genes whose products are plasma proteins directly involved in microbe immobilization or killing. The transcripts of Tc-proPOs, lysozyme1 or lysozyme 4 did not significantly change when compared with the controls, whereas those of $T c$-lysozyme 2 and 3 increased remarkably (Figure 8). The most dramatic increase in mRNA levels occurred in the AMP group of effector molecules, including Tc-attacin2, cecropin3, coleoptericin1, defensin1, and defensin2.

Cluster analysis of the expression patterns has revealed several trends of the transcriptional control of these immune genes. Buffer injected and uninjured adults form one cluster with the lowest mRNA levels, whereas $E$. coli- and $S$. cerevisiae-treated insects have the next higher level of overall gene expression (Figure 8). The yeast-injected beetles, instead of grouping with $E$. coli-treated insects, are found in the same cluster with $C$. albicans-challenged adults. Interestingly, immune responses toward the opportunistic fungal pathogen are greater than those toward $S$. cerevisiae, an environmental non-pathogen present in the diet. The responses toward $M$. luteus and C. albicans were significantly stronger than those towards $E$. coli, implying that the Toll pathway triggered by the Gram-positive bacteria and filamentous fungi more effectively up-regulated target gene expression than the IMD pathway did, which may be activated by the Gram-negative bacterial infection (Figure 5).

\section{Conclusion}

Through this comparative genome analysis, we have provided evidence in the red flour beetle for the functional conservation of intracellular immune signaling pathways (Toll, IMD and JAK/SAT) and for the evolutionary diversification of over 20 families of proteins (for example, PGRPs, clip-domain proteins, serpins, Toll-related receptors, antimicrobial proteins and scavenger receptors) involved in different mechanisms of insect defense against infection. The observed differences in conservation are likely related to distinct needs for specific molecular interactions and changes in microorganisms encountered by the host insects. For instance, Drosophila Myd88, Tube, Pelle, Pellino and TRAF, which form a macromolecular complex with the Toll/interleukin 1 receptor domain (Figure 5), have 1:1 orthologs in Anopheles, Apis and Tribolium. In contrast, family expansion and sequence divergence in the PGRP and AMP families are perhaps important for specific recognition and effective elimination of evolving pathogens.

The summary of putative immune gene counts, families and functions (Additional data file 11) suggests that $T$. castaneum has a more general defense than A. gambiae does. While this system is critical for the survival of this beetle, we are unclear whether or not it correlates with the prosperity of coleopteran insects. Drastic lineage-specific expansions seem sporadic and, in most cases, Tribolium paralog counts are lower than 


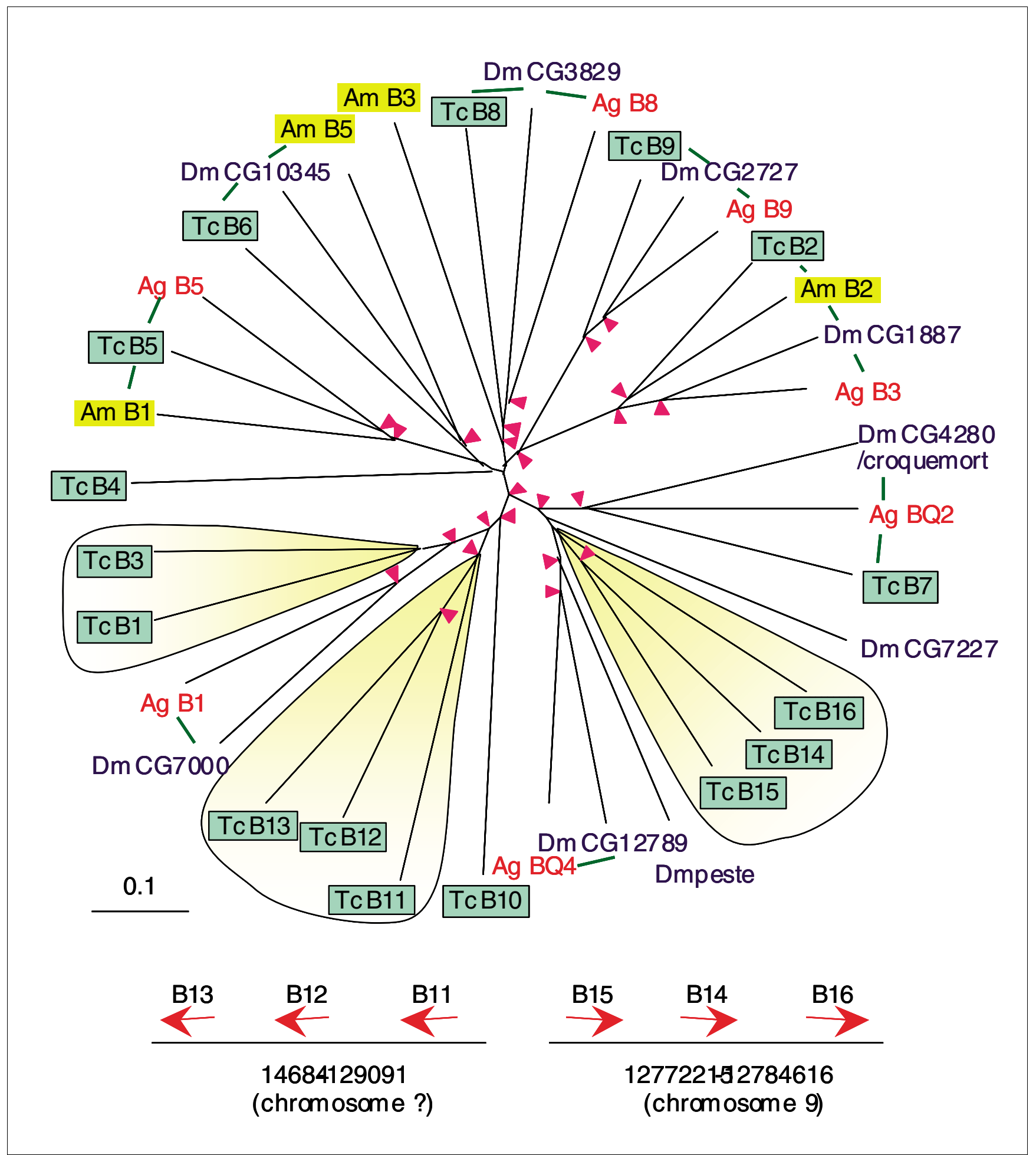

Figure 7

Phylogenetic analysis of class B SRs (SR-Bs). The aligned central parts, including the CD36 domain, of sixteen Tribolium (Tc), eight Drosophila (Dm), eight Anopheles $(A g)$ and three Apis (Am) SR-B sequences are used for building the unrooted tree (upper panel). For simplicity, the other members of class B SRs from Drosophila (seven) and Anopheles (four) are not included in this analysis. Lineage-specific expansion (shaded yellow) is confirmed in the complete tree that includes all SR-Bs from the four species. The expansion is consistent with their chromosomal locations (lower panel). Pink arrowheads indicate nodes with bootstrap values exceeding 800 (from I,000 trials), whereas green bars connect the putative orthologs with I:I, I:I:I or I:I:I:I relationship. 


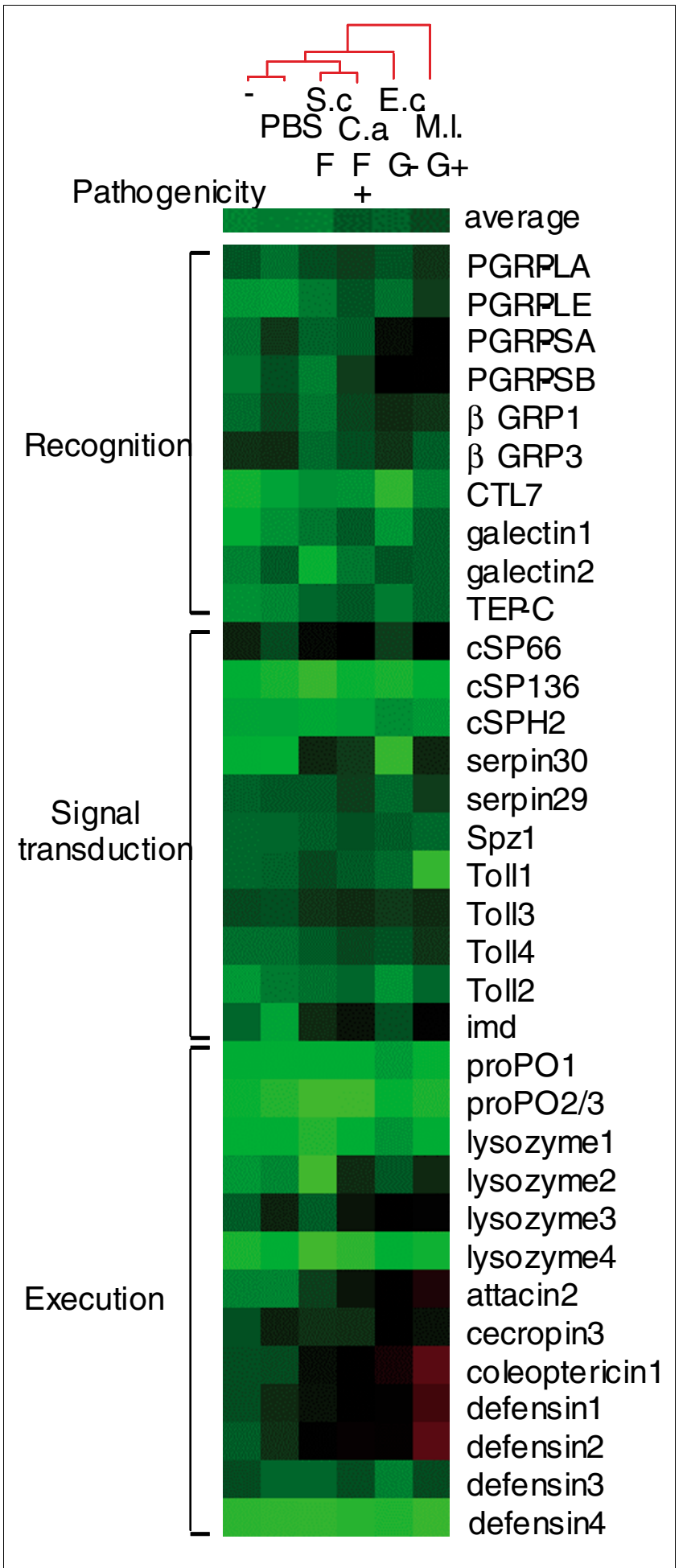

Figure 8

Real-time PCR analysis of expression of Tribolium immunity-related genes in adults $24 \mathrm{~h}$ after injections of M. luteus (M.I.), E. coli (E.c.), C. albicans (C.a.), S. cerevisiae (S.c.), or phosphate-buffered saline (PBS). Uninjured insects (-) were used as another negative control. With green, black and red colors representing low, intermediate and high transcript levels, respectively, relative mRNA abundances were used to cluster samples by average-linker clustering. those of Anopheles or Drosophila (but are considerably higher than of Apis). The only exceptions are the clip-domain SP/SPH and serpin families: 48,41 and 37 proteinase-related genes and 31, 14 and 28 inhibitor genes are present in the beetle, mosquito and flies, respectively. Because clip-domain SPs are often regulated by serpins, positive selection may have played a role in the converted evolution of both families and in the maintenance of homeostasis.

This comparative analysis has also uncovered interesting genes and gene families for future research. For instance, the existence of a 1:1 ortholog of Drosophila PGRP-LE in Tribolium (but not in Anopheles or Apis) may allow us to test whether or not TcPGRP-LE has a similar function. It can be interesting to explore the molecular mechanisms and evolutionary pathways of the large serpin and SP gene clusters in the beetle. The presence of TcToll-1 through -4 and subtle changes in their mRNA levels after immune challenges call for detailed analysis of their transcriptional regulation and physiological functions. Of course, the proposed extracellular and intracellular signaling pathways need to be tested, even though we have confidence in their general structures. The possible AMP function of Tc11324, which contains two whey acidic protein motifs, needs to be established experimentally.

It is noteworthy that the functions of Tribolium immunityrelated genes are mostly assumed based on sequence similarity to studied proteins in Drosophila or other insect species. Functional analyses using the strong reverse genetic techniques available in Tribolium are necessary to test the hypotheses. Nevertheless, the framework of information established in this work should help clarify immune functions in an important agricultural pest from the most diverse insect order and a species that can serve as a tractable model for an innate immune system more generally.

\section{Materials and methods}

\section{Database search and sequence annotation}

Known defense proteins from other insects were used as queries to perform BLASTP searches of Tcastaneum Glean Predictions (2005.10.11) [63]. Protein sequences with E-values lower than 0.1 were listed, and every $5^{\text {th }}$ sequence was retrieved for use as a query for another round of search. Based on the combined lists, respective protein sequences were retrieved, compiled in the order of ascending E-values, and improved by two methods. Firstly, Tcastaneum ESTs (2005.9.20) at the same HGSC site were searched with the corresponding nucleotide sequences to identify possible cDNA clones. The EST sequences were assembled using CAP3 [64] and the resulting contigs were used in pairwise comparison [65] to validate the gene predictions. Secondly, retrieved protein sequences were analyzed by CDART [66], PROSITE [67], and SMART [68] to detect conserved domain structures required for specific functions. Necessary changes were made after each step to improve the original predictions. Chromo- 
somal location and exon-intron boundaries for each annotated sequence were acquired from Genboree [69]. To locate orthologs not identified by BLASTP, Tribolium Genome Assembly 2.0 [70] was searched using TBLASTN. The hits detected were analyzed using multiple gene prediction tools Genescan and Genemark [71,72]. All curated sequences then were deposited in the annotation database [73] as a part of Tribolium Genome Assembly 2.o.

\section{Phylogenetic analyses}

Unless otherwise specified, full-length Tribolium sequences were aligned with their homologs from other insects, including D. melanogaster, A. gambiae and A. mellifera. The sequences were retrieved from NCBI [74], Flybase [75], or Ensembl [76]. Multiple sequence alignments were carried out using ClustalX [77] and Blosum series of weight matrices [78]. Phylogenetic trees were constructed based on algorithm of neighbor-joining using PHYLIP [79] or maximum-parsimony using PAUP [8o]. The divergence time of Tc-proPO2 and proPO3 were calculated using the rate of $1.7 \times 10^{-8}$ synonymous substitutions/nucleotide/year derived from the Drosophila species [54].

\section{Gene expression analysis}

To study pathogen-induced gene expression, adult red flour beetles (approximately 240 per group) were pricked at the ventral thorax with needles dipped in sterile phosphate-buffered saline or the buffer containing concentrated live E. coli, $M$. luteus, C. albicans or $S$. cerevisiae cells. Uninjured and aseptically injured insects were employed as controls. Total RNA samples were extracted from the control and challenged insects (approximately 160 per group) $24 \mathrm{~h}$ later, using Micro-to-mid RNA Purification System (Invitrogen, Carlsbad, CA, USA). After DNA removal, each RNA sample (1.0-3.4 $\mu \mathrm{g})$, oligo(dT) $(0.5 \mu \mathrm{g}, 1 \mu \mathrm{l})$ and dNTPs (10 mM each, $1 \mu \mathrm{l}$ ) were mixed with diethyl pyrocarbonate-treated $\mathrm{H}_{2} \mathrm{O}$ in a final volume of $12 \mu \mathrm{l}$, and denatured at $65^{\circ} \mathrm{C}$ for 5 minutes. First strand cDNA was synthesized for 50 minutes at $42^{\circ} \mathrm{C}$ using SuperScript Reverse Transcriptase (200 U/ $\mu$ l, $1 \mu \mathrm{l}$; Invitrogen) mixed with $5 \times$ buffer $(4 \mu \mathrm{l})$, o.1 M dithiothreitol (2 $\mu \mathrm{l}$ ), RNase OUT (40 U/ $\mu \mathrm{l}, 1 \mu \mathrm{l}$; Invitrogen) and the denatured RNA sample $(12 \mu \mathrm{l})$. Specific primer pairs were designed for a total of 35 immunity-related genes (Additional data file 12) using Primer 3 [81] with annealing temperatures of 59.5$60.5^{\circ} \mathrm{C}$ and expected product sizes of $80-150 \mathrm{bp}$. Each primer pair was located in adjacent exons flanking an intron. Realtime PCR was performed in parallel reactions on 96-well microtiter plates using Taq DNA polymerase $(1 \mathrm{U}$; Roche Applied Sciences, Indianapolis, IN, USA), $1 \times$ buffer, $1 \mathrm{mM}$ dNTP mix, $2 \mathrm{mM} \mathrm{MgCl}_{2}$, $0.2 \mu \mathrm{M}$ primers, $1 \times$ SYBR-Green I dye (Applied Biosystems, Foster City, CA, USA) and $10 \mathrm{nM}$ fluorescein. Amplifications were enacted on an iCycler thermal cycler (Bio-Rad, Hercules, CA, USA) with a profile of $95^{\circ} \mathrm{C}$ for 5 minutes followed by 40 cycles of $94^{\circ} \mathrm{C}$ for $20 \mathrm{~s}$, $60^{\circ} \mathrm{C}$ for $30 \mathrm{~s}, 72^{\circ} \mathrm{C}$ for $60 \mathrm{~s}$ and $78^{\circ} \mathrm{C}$ for $20 \mathrm{~s}$ [82]. SYBR green fluorescence was measured during the $78^{\circ} \mathrm{C}$ step in each cycle and the cycle numbers for each target and control gene were recorded when the fluorescence passed a predetermined threshold. Proper dissociation and correct size of the products were examined by melting curve analysis and agarose gel electrophoresis, respectively. The real-time PCR was repeated twice and, in each of the three experimental replicates, the transcripts were normalized relative to the levels of Tribolium ribosomal protein S3. Averaged transcript abundance values $\left(\mathrm{Ct}_{\text {control }}-\mathrm{Ct}_{\text {target }}\right)$ were then compared across genes and samples using average-linking clustering (Cluster 3.0) and visualized using TreeView [83].

\section{Abbreviations}

$\beta$ GRP, $\beta$-1,3-glucan-recognition protein; AMP, antimicrobial peptide; CTL, C-type lectin; FREP, fibrinogen-related protein; GNBP, Gram-negative binding protein; GTX, glutathione oxidase; PGRP, peptidoglycan recognition protein; PPAF, proPO activating factor; proPO, prophenoloxidase; RNS, reactive nitrogen species; ROS, reactive oxygen species; SOD, superoxide dismutase; SP, serine proteinase; SPH, noncatalytic serine proteinase homolog; SR, scavenger receptor; TEP, thioester-containing protein; TPX, peroxiredoxin.

\section{Authors' contributions}

Zhen Zou: study design; data collection, analysis and deposition; annotation of clip-domain SPs/SPHs, serpins, spätzles, SRs and others; Toll and Imd pathways. Jay Evans: RT-PCR analysis; GNBPs and PGRPs. Zhiqiang Lu: C-type lectins, galectins, TEPs and JAK/STAT pathway. Picheng Zhao: Tolllike receptors, caspases and ROS/RNS production. Michael Williams and Dan Hultmark: FREPs, Nimrods, PGRPs and cecropins. Charles Hetru and Niranji Sumathipala: antimicrobial peptides and lysozymes. Haobo Jiang: study design; data analysis and interpretation; annotation of clip-domain SPs/SPHs; manuscript writing.

\section{Additional data files}

The following additional data are available with the online version of this paper. Additional data file 1 is a table listing immunity-related genes in T. castaneum. Additional data file 2 is a figure showing sequence alignments of $\beta$ GRPs and GNBPs. Additional data file 3 is a figure showing sequence alignments of CTLs. Additional data file 4 is a figure showing sequence alignments of galectins. Additional data file 5 is a figure showing sequence alignments of FREPs. Additional data file 6 is a figure showing sequence alignments of TEPs. Additional data file 7 is a figure showing sequence alignments of Spätzle-related proteins. Additional data file 8 is a figure showing sequence alignments of proPOs. Additional data file 9 is a figure showing sequences of GTX, SOD and TPX. Additional data file 10 is a figure showing sequence alignments of lysozymes. Additional data file 11 is a table listing functions, families, and counts of putative defense proteins from $D$. mel- 
anogaster, A. gambiae, A. mellifera and T. castaneum. Additional data file 12 is a table listing oligonucleotide primers used in expression analysis by real-time PCR.

\section{Acknowledgements}

We greatly appreciate our colleagues in the Tribolium genome sequence consortium for the gene prediction. Dr Thomas Phillips kindly provided the insects for immune challenges and RT-PCR experiments. We would also like to thank Drs Ulrich Melcher, Jack Dillwith, and Maureen Gorman for their helpful comments on the manuscript. This work was supported by the National Institutes of Health Grants GM58634 (to HJ). The article was approved for publication by the Director of Oklahoma Agricultural Experimental Station and supported in part under project OKLO2450.

\section{References}

I. Wade MJ, Chang NW: Increased male-fertility in Tribolium confusum beetles after infection with the intracellular parasite Wolbachia. Nature 1995, 373:72-74.

2. Blaser M, Schmid-Hempel P: Determinants of virulence for the parasite Nosema whitei in its host Tribolium castaneum. J Invertebr Pathol 2005, 89:25I-257.

3. Zhong D, Pai A, Yan G: Costly resistance to parasitism: evidence from simultaneous quantitative trait loci mapping for resistance and fitness in Tribolium castaneum. Genetics 2005, 169:2127-2135

4. Moret $Y$ : "Trans-generational immune priming": specific enhancement of the antimicrobial immune response in the mealworm beetle, Tenebrio molitor. Proc Biol Sci 2006, 273: I399-1405.

5. Arakane Y, Muthukrishnan S, Beeman RW, Kanost MR, Kramer KJ: Laccase 2 is the phenoloxidase gene required for beetle cuticle tanning. Proc Natl Acad Sci USA 2005, I 02: I I 337- I I 342.

6. Lemaitre B, Hoffmann J: The host defense of Drosophila melanogaster. Annu Rev Immunol 2007, 25:697-743.

7. Christophides GK, Vlachou D, Kafatos FC: Comparative and functional genomics of the innate immune system in the malaria vector Anopheles gambiae. Immunolog Rev 2004, I 98: I27-I 48.

8. Brown SJ, Denell RE, Beeman RW: Beetling around the genome. Genetical Res 2003, 82: I55-I6I.

9. Iwanaga $S$, Lee BL: Recent advances in the innate immunity of invertebrate animals. I Biochem Mol Biol 2005, 38: I 28-I 50.

10. Barbault F, Landon C, Guenneugues M, Meyer JP, Schott V, Dimarcq J, Vovelle F: Solution structure of Alo-3: a new knottin-type antifungal peptide from the insect Acrocinus longimanus. Biochemistry 2003, 42: |4434-|4442.

II. Saito A, Ueda K, Imamura M, Atsumi S, Tabunoki H, Miura N, Watanabe $A$, Kitami $M$, Sato R: Purification and cDNA cloning of a cecropin from the longicorn beetle, Acalolepta luxuriosa. Comparative Biochem Physiol B Biochem Mol Biol 2005, I 42:3 I7-323.

12. Bulet P, Cociancich S, Dimarcq JL, Lambert J, Reichhart JM, Hoffmann $D$, Hetru C, Hoffmann JA: Insect immunity. Isolation from a coleopteran insect of a novel inducible antibacterial peptide and of new members of the insect defensin family. J Biol Chem | 99|, 266:24520-24525.

13. Sagisaka A, Tanaka H, Furukawa S, Yamakawa M: Characterization of a homologue of the Rel/NF-kappaB transcription factor from a beetle, Allomyrina dichotoma. Biochim Biophys Acta 2004, 1678:85-93.

14. Kim MS, Baek MJ, Lee MH, Park JW, Lee SY, Soderhall K, Lee BL: A new easter-type serine protease cleaves a masquerade-like protein during prophenoloxidase activation in Holotrichia diomphalia larvae. J Biol Chem 2002, 277:39999-40004.

15. Kwon TH, Kim MS, Choi HW, Joo CH, Cho MY, Lee BL: A masquerade-like serine proteinase homologue is necessary for phenoloxidase activity in the coleopteran insect, Holotrichia diomphalia larvae. Eur J Biochem 2000, 267:6I88-6I96.

16. Lee SY, Kwon TH, Hyun JH, Choi JS, Kawabata SI, Iwanaga S, Lee BL: In vitro activation of pro-phenol-oxidase by two kinds of prophenol-oxidase-activating factors isolated from hemolymph of coleopteran, Holotrichia diomphalia larvae. Eur J Biochem 1998, 254:50-57.

I7. Zhao M, Soderhall I, Park JW, Ma YG, Osaki T, Ha NC, Wu CF, Sod- erhall $\mathrm{K}$, Lee BL: A novel 43-kDa protein as a negative regulatory component of phenoloxidase-induced melanin synthesis. J Biol Chem 2005, 280:24744-2475I.

18. Evans JD, Aronstein K, Chen YP, Hetru C, Imler JL, Jiang H, Kanost M, Thompson GJ, Zou Z, Hultmark D: Immune pathways and defence mechanisms in honey bees Apis mellifera. Insect Mol Biol 2006, I 5:645-656.

19. Kanost MR, Jiang HB, Yu XQ: Innate immune responses of a lepidopteran insect, Manduca sexta. Immunol Rev 2004, 198:97- 05.

20. Steiner H: Peptidoglycan recognition proteins: on and off switches for innate immunity. Immunol Rev 2004, 198:83-96.

21. Kim MS, Byun MJ, Oh BH: Crystal structure of peptidoglycan recognition protein LB from Drosophila melanogaster. Nat Immunol 2003, 4:787-793.

22. Mellroth $\mathrm{P}, \mathrm{Karlsson}$ J, Steiner $\mathrm{H}$ : A scavenger function for a Drosophila peptidoglycan recognition protein. J Biol Chem 2003, 278:7059-7064.

23. Jiang $H B, M a C C$, Lu ZQ, Kanost MR: $\beta$-I,3-Glucan recognition protein-2 (beta GRP-2) from Manduca sexta: an acute-phase protein that binds beta-I,3-glucan and lipoteichoic acid to aggregate fungi and bacteria and stimulate prophenoloxidase activation. Insect Biochem Mol Biol 2004, 34:89-I00.

24. Ochiai M, Ashida M: A pattern-recognition protein for beta-I, 3 glucan - The binding domain and the cDNA cloning of betaI,3-glucan recognition protein from the silkworm, Bombyx mori. J Biol Chem 2000, 275:4995-5002.

25. Wang $\mathrm{Y}$, Jiang $\mathrm{H}$ : Interaction of beta- I,3-glucan with its recognition protein activates hemolymph proteinase 14 , an initiation enzyme of the prophenoloxidase activation system in Manduca sexta. J Biol Chem 2006, 28I:927I-9278.

26. Vasta GR, Quesenberry M, Ahmed H, O'Leary N: C-type lectins and galectins mediate innate and adaptive immune functions: their roles in the complement activation pathway. Dev Comparative Immunol I999, 23:40 I-420.

27. Koizumi N, Imamura M, Kadotani T, Yaoi K, Iwahana H, Sato R: The lipopolysaccharide-binding protein participating in hemocyte nodule formation in the silkworm Bombyx mori is a novel member of the $C$-type lectin superfamily with two different tandem carbohydrate-recognition domains. FEBS Lett 1999, 443: 139-|43.

28. $\mathrm{Yu} X \mathrm{X}, \mathrm{Kanost} M \mathrm{R}$ : Immulectin-2, a lipopolysaccharide-specific lectin from an insect, Manduca sexta, is induced in response to gram-negative bacteria. J Biol Chem 2000, 275:37373-3738I.

29. Christophides GK, Zdobnov E, Barillas-Mury C, Birney E, Blandin S, Blass C, Brey PT, Collins FH, Danielli A, Dimopoulos G, et al.: Immunity-related genes and gene families in Anopheles gambiae. Science 2002, 298: I59-I65.

30. Tanji T, Ohashi-Kobayashi A, Natori S: Participation of a galactose-specific C-type lectin in Drosophila immunity. Biochem J 2006, 396: I27-I38

3I. Kamhawi S, Ramalho-Ortigao M, Pham VM, Kumar S, Lawyer PG, Turco S], Barillas-Mury C, Sacks DL, Valenzuela JG: A role for insect galectins in parasite survival. Cell 2004, I I 9:329-34 I.

32. Fujita T, Endo Y, Nonaka M: Primitive complement system recognition and activation. Mol Immunol 2004, 4I: I03-III.

33. Litman GW, Cannon JP, Dishaw LJ: Reconstructing immune phylogeny: New perspectives. Nat Rev Immunol 2005, 5:866-879.

34. Gokudan S, Muta T, Tsuda R, Koori K, Kawahara T, Seki N, Mizunoe Y, Wai SN, Iwanaga S, Kawabata S: Horseshoe crab acetyl grouprecognizing lectins involved in innate immunity are structurally related to fibrinogen. Proc Natl Acad Sci USA 1999, 96: I0086-1009I.

35. Schroder HC, Ushijima H, Krasko A, Gamulin V, Thakur NL, DiehlSeifert B, Muller IM, Muller WEG: Emergence and disappearance of an immune molecule, an antimicrobial lectin, in basal Metazoa - A tachylectin-related protein in the sponge Suberites domuncula. J Biol Chem 2003, 278:328I 0-328I7.

36. Wang X, Rocheleau TA, Fuchs JF, Hillyer JF, Chen CC, Christensen BM: A novel lectin with a fibrinogen-like domain and its potential involvement in the innate immune response of Armigeres subalbatus against bacteria. Insect Mol Biol 2004, I 3:273-282

37. Morisada T, Kubota Y, Urano T, Suda T, Oike Y: Angiopoietins and angiopoietin-like proteins in angiogenesis. Endothelium J Endothelial Cell Res 2006, I 3:71-79.

38. Mok LP, Qin TL, Bardot B, LeComte M, Homayouni A, Ahimou F, Wesley $C$ : Delta activity independent of its activity as a ligand 
of Notch. BMC Dev Biol 2005, 5:6.

39. Williams MJ: Drosophila hemopoiesis and cellular immunity. J Immunol 2007, 178:47| I-47|6.

40. Lagueux M, Perrodou E, Levashina EA, Capovilla M, Hoffmann JA: Constitutive expression of a complement-like protein in Toll and JAK gain-of-function mutants of Drosophila. Proc Natl Acad Sci USA 2000, 97: | | 427- I |432.

4I. Ross J, Jiang H, Kanost MR, Wang Y: Serine proteases and their homologs in the Drosophila melanogaster genome: an initial analysis of sequence conservation and phylogenetic relationships. Gene 2003, 304:II7-|3|.

42. Zou Z, Lopez DL, Kanost MR, Evans JD, Jiang HB: Comparative analysis of serine protease-related genes in the honey bee genome: possible involvement in embryonic development and innate immunity. Insect $\mathrm{Mol} \mathrm{Biol} 2006$, I5:603-6|4.

43. Jiang $H B$, Kanost MR: The clip-domain family of serine proteinases in arthropods. Insect Biochem Mol Biol 2000, 30:95- 105.

44. Tang H, Kambris Z, Lemaitre B, Hashimoto C: Two proteases defining a melanization cascade in the immune system of Drosophila. J Biol Chem 2006, 28 I:28097-28I 04.

45. Jang IH, Chosa N, Kim SH, Nam HJ, Lemaitre B, Ochiai M, Kambris Z, Brun S, Hashimoto C, Ashida M, et al.: A Spatzle-processing enzyme required for toll signaling activation in Drosophila innate immunity. Dev Cell 2006, 10:45-55.

46. Ligoxygakis P, Pelte N, Hoffmann JA, Reichhart JM: Activation of Drosophila Toll during fungal infection by a blood serine protease. Science 2002, 297: I I4-II6.

47. Kanost MR: Serine proteinase inhibitors in arthropod immunity. Dev Comparative Immunol 1999, 23:29I-30I.

48. Hultmark D: Drosophila immunity: paths and patterns. Curr Opin Immunol 2003, 15:12-19.

49. Miller SI, Ernst RK, Bader MW: LPS, TLR4 and infectious disease diversity. Nat Rev Microbiol 2005, 3:36-46.

50. Dong Y, Aguilar R, Xi Z, Warr E, Mongin E, Dimopoulos G: Anopheles gambiae immune responses to human and rodent Plasmodium parasite species. PLOS pathogens 2006, 2:e52.

5I. Wang LH, Ligoxygakis P: Pathogen recognition and signalling in the Drosophila innate immune response. Immunobiology 2006, 2I I:25I-26I.

52. Agaisse H, Perrimon N: The roles of JAK/STAT signaling in Drosophila immune responses. Immunol Rev 2004, 198:72-82.

53. Nappi AJ, Christensen BM: Melanogenesis and associated cytotoxic reactions: Applications to insect innate immunity. Insect Biochem Mol Biol 2005, 35:443-459.

54. Rowan RG, Hunt JA: Rates of DNA change and phylogeny from the DNA-sequences of the alcohol-dehydrogenase gene for five closely related species of Hawaiian Drosophila. Mol Biol Evol I99I, 8:49-70.

55. Kumar S, Barillas-Mury C: Ookinete-induced midgut peroxidases detonate the time bomb in anopheline mosquitoes. Insect Biochem Mol Biol 2005, 35:72 I-727.

56. Dejong RJ, Miller LM, Molina-Cruz A, Gupta L, Kumar S, Barillas-Mury $C$ : Reactive oxygen species detoxification by catalase is a major determinant of fecundity in the mosquito Anopheles gambiae. Proc Natl Acad Sci USA 2007, 104:2121-2I 26.

57. Bulet $P$, Stocklin R, Menin L: Anti-microbial peptides: from invertebrates to vertebrates. Immunol Rev 2004, 198:169-184.

58. Hedengren M, Borge K, Hultmark D: Expression and evolution of the Drosophila Attacin/Diptericin gene family. Biochem Biophys Res Commun 2000, 279:574-58I.

59. Hagiwara K, Kikuchi T, Endo Y, Huqun, Usui K, Takahashi M, Shibata $\mathrm{N}$, Kusakabe T, Xin H, Hoshi S, et al: Mouse SWAMI and SWAM2 are antibacterial proteins composed of a single whey acidic protein motif. J Immunol 2003, I 70:1973-1979.

60. Lavine MD, Strand MR: Insect hemocytes and their role in immunity. Insect Biochem Mol Biol 2002, 32:1295-1309.

61. Cherry S, Silverman N: Host-pathogen interactions in Drosophila: new tricks from an old friend. Nat Immunol 2006, 7:911-917.

62. Kurucz E, Markus R, Zsamboki J, Folkl-Medzihradszky K, Darula Z, Vilmos P, Udvardy A, Krausz I, Lukacsovich T, Gateff E, et al.: Nimrod, a putative phagocytosis receptor with EGF repeats in Drosophila plasmatocytes. Curr Biol 2007, 17:649-654.

63. Tcastaneum Glean Predictions [http://www.hgsc.bcm.tmc.edu/ blast.hgsc?organism $=13$ ]

64. Huang $X Q$, Madan A: CAP3: A DNA sequence assembly program. Genome Res 1999, 9:868-877.

65. BLAST 2 Sequences [http://www.ncbi.nlm.nih.gov/blast/bl2seq/ wblast2.cgi]

66. CDART [http://www.ncbi.nlm.nih.gov/structure/lexington/lexing ton.cgi?cmd=rps]

67. PROSITE [http://us.expasy.org/prosite/]

68. SMART [http://smart.embl-heidelberg.de/smart/set_mode.cgi?nor $\mathrm{mal}=\mathrm{I}]$

69. Genboree [http://www.genboree.org/java-bin/login.jsp]

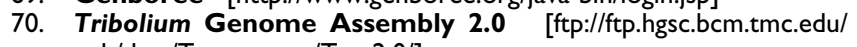
$\mathrm{pub} / \mathrm{data} / \mathrm{Tcastaneum} / \mathrm{Tcas} 2.0 /]$

71. GENSCAN [http://genes.mit.edu/GENSCAN.html]

72. GeneMark [http://opal.biology.gatech.edu/GeneMark/eukhmm.cgi]

73. Annotation Database [http://annotation.hgsc.bcm.tme.edu/]

74. NCBI [http://www.ncbi.nlm.nih.gov/]

75. Flybase [http://flybase.bio.indiana.edu/]

76. Ensembl [http://www.ensembl.org/]

77. Chenna R, Sugawara H, Koike T, Lopez R, Gibson TJ, Higgins DG, Thompson JD: Multiple sequence alignment with the Clustal series of programs. Nucleic Acids Res 2003, 3 I:3497-3500.

78. Henikoff S, Henikoff JG: Amino acid substitution matrices from protein blocks. Proc Natl Acad Sci USA 1992, 89:10915-10919.

79. PHYLIP [http://evolution.genetics.washington.edu/phylip.html]

80. PAUP [http://paup.csit.fsu.edu/]

81. Primer 3 [http://frodo.wi.mit.edu/cgi-bin/primer3/
3 primer3_www.cgi]

82. Evans JD: Beepath: An ordered quantitative-PCR array for exploring honey bee immunity and disease. J Invertebrate Pathol 2006, 93:135-139.

83. TreeView [http://rana.lbl.gov/EisenSoftware.htm] 